\title{
The Effects of Propofol on the Human Blood Brain Barrier
}

\author{
Kirsten A. Lewis, Jason M. Hughes, and Scott G. Canfield \\ Department of Cellular and Integrative Physiology, Indiana University School of \\ Medicine-Terre Haute
}

\section{Background and Hypothesis:}

Recently, the safety of repeated and lengthy anesthesia in young children has been called into question. Previous studies have shown propofol, an anesthetic, can diminish blood-brain barrier (BBB) properties. However, the underlying cellular mechanisms are relatively unknown. The BBB is critical in ensuring that potentially harmful circulating factors are impermeable to the brain. Previous animal studies models have shown that propofol increases the levels of matrix metalloproteinases (MMPs), which have independently been shown to degrade the extracellular matrix and breakdown tight junctions, a critical component of the BBB. Hypothesis: Propofol exposure to a human induced pluripotent stem cell (iPSC)-derived BBB model increases MMP activity ultimately contributing to a leaky barrier phenotype.

\section{Experimental Design or Project Methods:}

This study utilized human iPSCs differentiated into brain microvascular endothelial cells (BMECs), the barrier forming cell type of the BBB. iPSC-derived BMECS were exposed to propofol $(50 \mu \mathrm{M})$ for three hours and barrier properties were monitored. Barrier tightness was monitored using trans-endothelial electrical resistance (TEER) and sodium fluorescein permeability assays. Tight junction localization was determined with immunocytochemistry. MMP activity was determined with SensoLyte ${ }^{\circledR}$ assay kits. To determine the role of MMPs, a broad spectrum MMP inhibitor, GM6001, was utilized and barrier properties were monitored.

\section{Results:}

Propofol treatment significantly reduced TEER and increased sodium fluorescein permeability, indicative of a leaky barrier. Propofol treatment increased levels of MMP-2 activity but not MMP-9 when compared to non-treated BMECs. Inhibition of MMPs by GM6001 prior to propofol treatment appeared to partially restore barrier integrity as monitored by sodium fluorescein permeability.

\section{Conclusion and Potential Impact:}

These results indicate that increased MMP-2 activity levels could be in part responsible for diminished BBB properties. Inhibition of MMPs protected barrier integrity from propofol treatment. A further understanding of the underlying mechanisms of anesthetic-induced damage can potentially improve anesthesia safety. 